
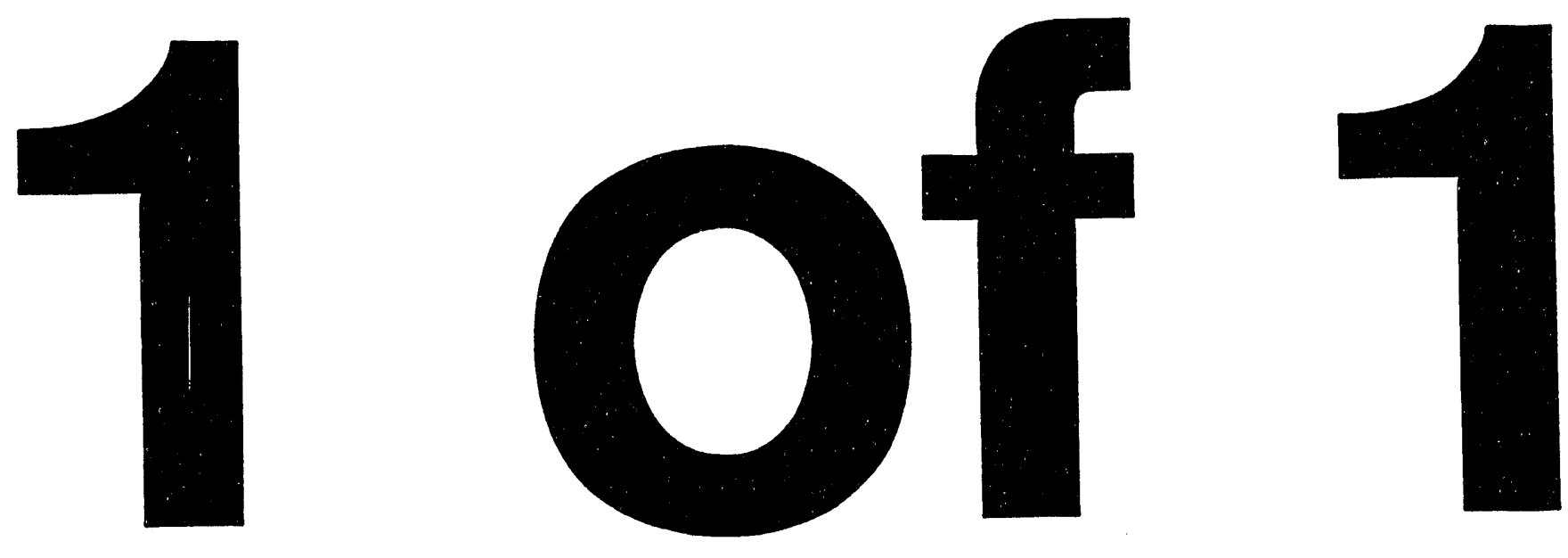


\title{
Conf $-940630--29$
}

UCRL-JC-115588

PREPRINT

\section{Target Alignment in the National Ignition Facility}

\author{
Charles S. Vann \\ Erlan S. Bliss \\ James E. Murray
}

This paper was prepared for submittal to the

Eleventh Topical Meeting on the

Technology of Fusion Energy

June 19-23, 1994

New Orleans, LA

June 6, 1994

This is a preprint of a paper intended for publication in a journal or proceedings. Since changes may be made betore publication, this preprint is made available with the understanding that it will not be cited or reproduced without the permission of the author. 


\section{DISCLAIMER}

This document was prepared as an account of work sponsored by an agency of the United States Government. Neither the United States Government, nor the University of California, nor any of their employees makes any warranty, express or implied, or assumes any legal liability or responsibility for the accuracy, completeness, or usefulness of any information, apparatus, product, or process disclosed, or represents that its use would not infringe privately owned rights. Reference herein to any specific commercial products, process, or service by trade name, trademark, manufacturer, or otherwise, does not necessarily constitute or imply its endorsement, recommendation, or favoring by the United States Government or the University of California. The views and opinions of authors expressed herein do not necessarily state or reflect those of the United States Government or the University of California, and shall not be used for advertising or product endorsement purposes. 


\section{TARGET ALIGNMENT IN THE NATIONAL IGNITION FACILITY}

$\begin{array}{lll}\text { Charles S. Vann } & \text { Erlan S. Bliss } & \text { James E. Murray } \\ \text { Lawrence Livermore National Laboratory Lawrence Livermore National Laboratory Lawrence Livermore National Laboratory } \\ \begin{array}{lll}\text { P.O. Box 808 (L-493) } & \text { P.O. Box } 808 \text { (L-463) } & \text { P.O. Box 808 (L-493) } \\ \text { Livermore, California 94551 } & \text { Livermore, California 94551 } & \text { Livermore, California 94551 } \\ \text { (510) 423-8201 } & \text { (510) } 422-5483 & \text { (510) 422-5481 }\end{array}\end{array}$

\begin{abstract}
Accurate placement of hundreds of focused laser beams on target is necessary to achieve success in the National Ignition Facility (NIF). The current system requirement is $\leq 7 \mu \mathrm{rad}$ error in output pointing and $\leq 1 \mathrm{~mm}$ error in focusing. To accommodate several system shots per day, a target alignment system must be able to align the target to chamber center, inject an alignment beam to represent each shot beam, and point and focus the alignment beams onto the target in about one hour. At Lawrence Livermore National Laboratory, we have developed a target alignment concept and built a prototype to validate the approach. The concept comprises three systems: the chamber center reference, target alignment sensor, and target alignment beams.
\end{abstract}

\section{INTRODUCTION}

The design for beam control is strongly driven by cost and performance considerations. The NIF laser has 192 beams, almost 20 times as many as its predecessor, Nova, yet the control and characterization of the beams must be accomplished at about the same cost (in FY95 dollars) and shot preparation time. Furthermore, the requirenıents for pointing beams on target are much more stringent:

- Point all beams at the target to $\leq 7 \mu \mathrm{rad} \mathrm{rms}$. This leads to typical values of $0.6 \mu \mathrm{rad}$ and $6 \mu \mathrm{m}$ for individual adjustment and stability requirements.

- Focus beams on target to a spot size of $\leq 500 \mu \mathrm{m}$. This requires correcting the laser wavefront to $\sim 25 \mu \mathrm{rad}$ and positioning the focusing lens to $\sim 1 \mathrm{~mm}$.

These challenging requirements imply certain alignment hardware and software design criteria:

Performance criteria:

- Automate functions to the maximum extent practical.

- Operate systems independently and in parallel.

- Complete tasks in $\leq 2 \mathrm{hr}$ for a higher shot rate and less alignment drift.

- Perform periodic calibration and validation of performance.
Cost-reduction criteria:

- Use commercial hardwarc and technology developments if possible.

- Minimize the number of remotely insertible components.

- Maxim:ze modularity to gain the cost benefits of volume production.

- Multiplex components to perform several functions for multiple beams.

- Simplify image-processing requirements.

The functional requirements and criteria were the inputs for totally new approaches in target alignment. The following sections describe the new target alignment tasks and the new concepts proposed to accomplish those tasks.

\section{TARGET CHAMBER CENTER REFERENCE SYSTEM}

Numerous diagnostic instruments are aligned to the target chamber center (TCC) to monitor target performance. To avoid realigning these instruments for each shot, the targets must also be positioned to the TCC. Since some of these instruments have fields of view less than $100 \mu \mathrm{m}$, the accuracy needed for target placement is a few micrometers. Consequently, a high-resolution system is required to define the TCC and to sense the location of targets (or fiducials) relative to it. The TCC will be established during initial setup by using a computer-aided theodolite system. Once established, the TCC will be permanently maintained by the chamber center referencing system, which views the position of any light-source fiducial in the vicinity of the TCC. With this system, devices having suitable lightsource fiducials can be repeatably and : curately inserted to the TCC.

The chamber center reference system consists of two simple imaging systems, as illustrated in Fig. 1. Each imaging system has a $30-\mathrm{cm}$-diameter positive lens just outside the chamber and a charge-coupled device (CCD) camera farther from the chamber to image a light source at the 'rCC onto the camera. With image processing, the resolution of the light-source position is better than $5 \mu \mathrm{m}$. 


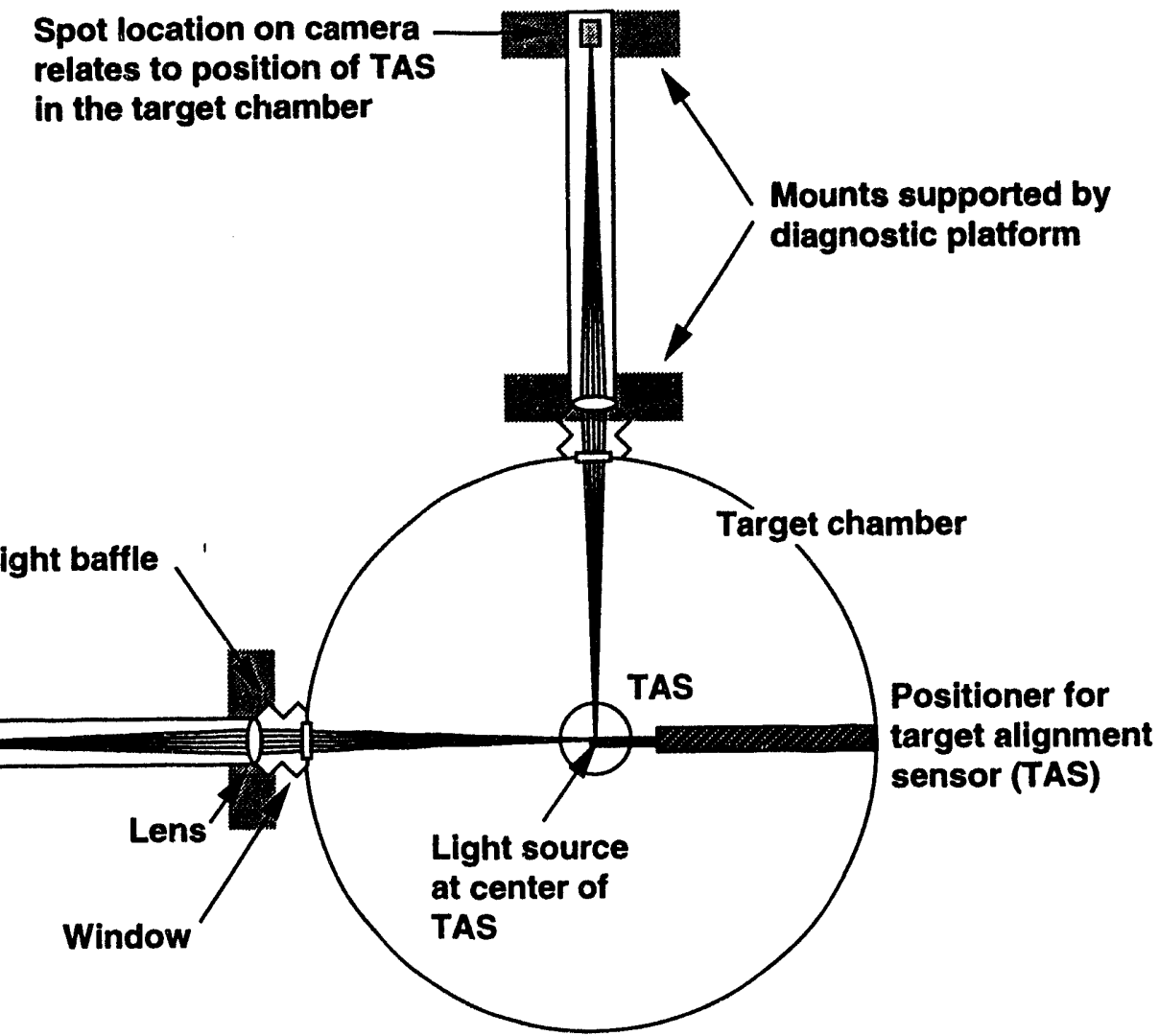

Figure 1. A two-channel imaging system defines the target chamber center (TCC).

The absolute position of the light source is not as important as the ability to place it repeatably, which is determined by the stability of the CCD camera and the lens. The lens and camera are analogous to cross hairs in an alignment scope. A single lens/camera system provides position information for two axes of translation. A second lens/camera system at an orthogonal angle provides information on the third translation axis.

Outside temperature changes, changes in ground water, curing of cement, and other effects may move the cameras and lenses relative to a reference frame external to the building. Likewise, the target diagnostics and target chamber would also move as a result of the same or different effects. Therefore, target diagnostics and the chamber center reference system will be supported on the same platform to minimize the motion between them.

\section{TARGET ALIGNMENT SENSOR}

The most direct and least expensive way to position beams on the target is to deploy a viewing instrument at the TCC because one device aligns all the beams. This instrument, the target alignment sensor (TAS), is our final alignment reference for all beams and the target (see Fig. 2). A motorized positioner inserts the TAS into the center of the chamber, where it provides a common reference to which the beams and target are positioned before each shot. Tilting the final transport mirror adjusts beam pointing on target, refocusing the final lens changes the beam spot size on target, and translating the target positioner corrects target position. The TAS cameras can simultaneously view the target and the beams as if beams were illuminating the target, but they do not actually reach it. This feature protects the target from preheating by alignment beams. Fiber-optic lighting sources enable combinations of low-level front and back target illumination for viewing. A fiducial on the TAS provides a reference for periodically checking the target diagnostics alignment. When alignment is complete, the TAS is removed to a protected location.

The TAS prototype shown in Fig. 3 was built to test the concept. A five-degree-of-freedom target positioner and three target alignment beams simulated the target chamber environment. The TAS was positioned with six motorized degrees of freedom, and two additional motors provided other necessary mobility. With the TAS, target positioner, and beam-alignment motors computer controlled, the system successfully performed autonated target and final beam-alignment functions. 


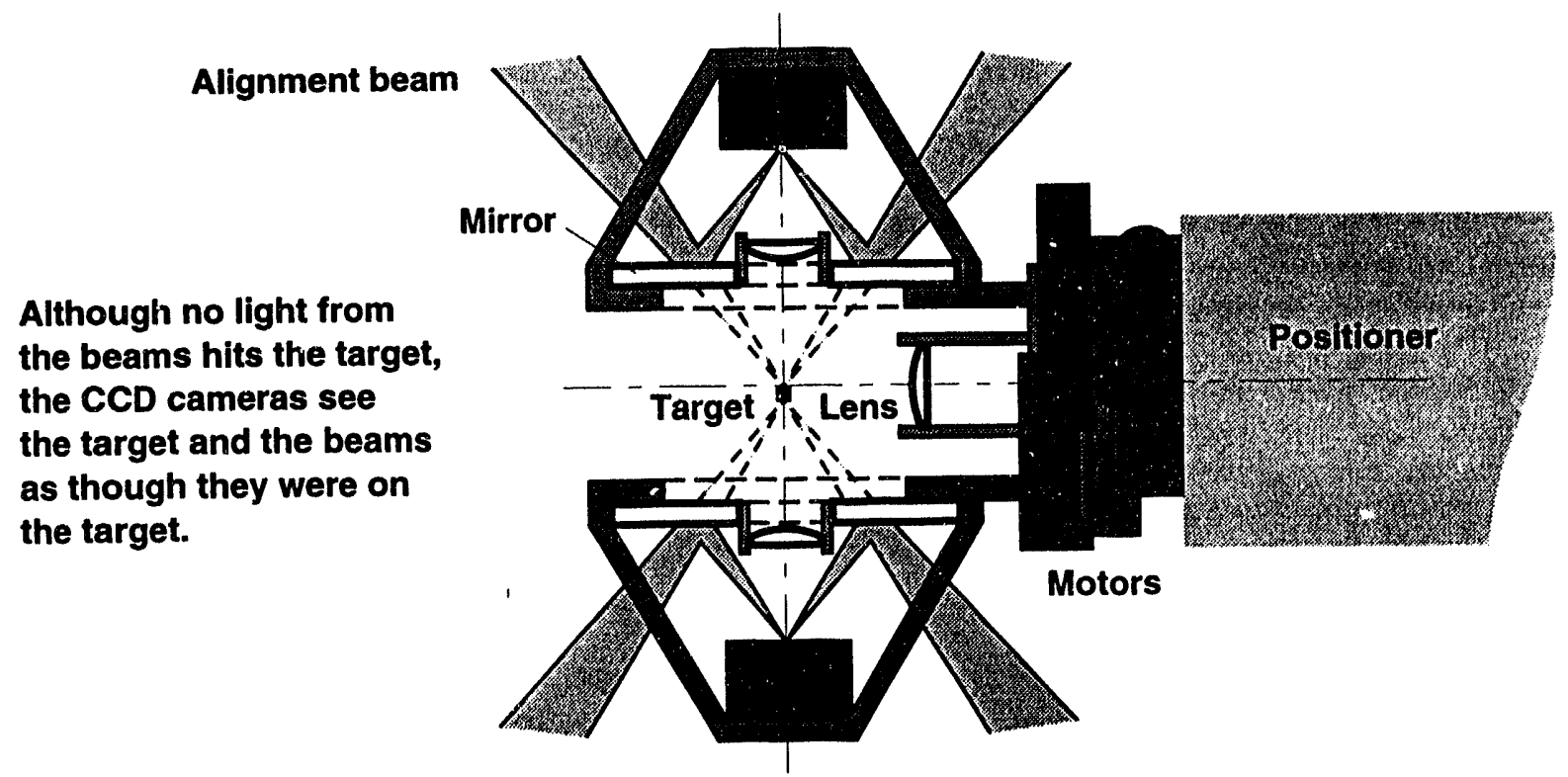

Figure 2. The beams and target are aligned to the target alignment sensor.

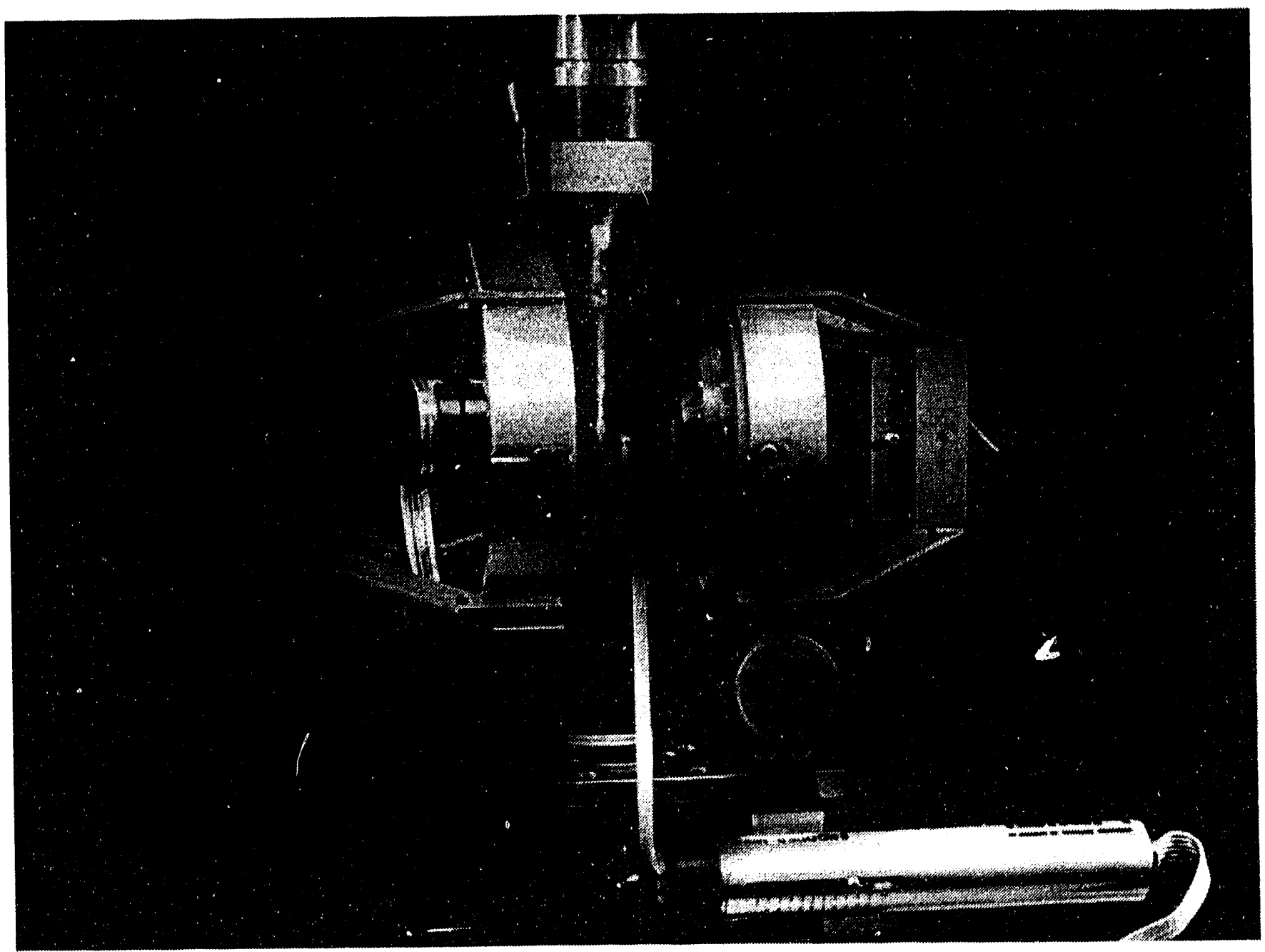

Figure 3. Prototype target alignment sensor (TAS). 
The chamber center reference system aids in returning the TAS to the same chamber position each time. Accurate repeatability minimizes the need for realigning the target diagnostics. A light source is fixed to a bar on the TAS that translates into the center of the TAS. The TAS cameras can verify that the light source is centered with respect to the TAS. When the TAS is inserted into the chamber, the chamber center referencing system senses the relative location of the light source (i.e., the TAS relative to the TCC). Feedback from the reference cameras is sent to a computer that calculates and sinds the necessary commands to place the TAS at the TCC. Since the target is subsequently aligned to the TAS center, this light source also represents the target location and provides a reference for aligning the target diagnostics.

\section{TARGET ALIGNMENT BEAMS}

The alignment beam power required by the TAS CCD cameras is only a few tens of nanowatts in each beam, so a low-power light source to represent the laser beam output is injected with an optical fiber at the pinhole plane of each transport spatial filter, as shown in Fig. 4. Except for the deviations due to dispersion that are discussed in the next paragraph, each alignment beam then follows the shotbeam path and permits adjustment of mirrors and lenses to establish the desired beam position and size on target. Since these beams do not pass through the laser, target alignment for the next shot can proceed independently of the alignment condition in the rest of the laser. This is an important capability because each time the laser is fired, its alignment condition and the properties of the laser alignment beams are affected for several hours while the optical components and their mounts cool.
In fact, the wavelength of the target alignment beams does not match either the operating wavelength of the laser $(1053 \mathrm{~nm})$ or the frequency-converted beam that illuminates the target $(351 \mathrm{~nm})$. Rather, it is set at an intermediate wavelength $(\sim 425 \mathrm{~nm})$ that results in an accurate relay of the pinhole plane in the final spatial filter to the target position. At this wavelength, the effects of dispersion in the spatial filter lens and final focus lens cancel, and the alignment beam focuses at the same distance from the focus lens as does the 351-nm shot beam. Lateral dispersion due to wedges in the delivery system does not cancel, however, so the intermediate-wavelength alignment source is injected with the appropriate lateral offset in the pinhole plane of the transport spatial filter (see Fig. 4). Frequency-doubled diode lasers are proposed for the light source, with large fiber-optic fanouts to produce up to 48 sources per laser. Four of these fanouts could supply all the target alignment beams for the entire system.

\section{ACKNOWLEDGMENTS}

Other contributors were Ray Ellis, Ken Moore, Mike Rushford, and Lynn Seppala. Work performed under the auspices of the U.S. Department of Energy by the Lawrence iivermore National Laboratory under Contract No. W-7405-Eng-48.

\section{REFERENCE}

1. National Ignition Facility Conceptual Design Report, Vol. 3, Lawrence Livermore National Laboratory, Livermore, CA, UCRL-PROP-117093 Vol 3 (1994).

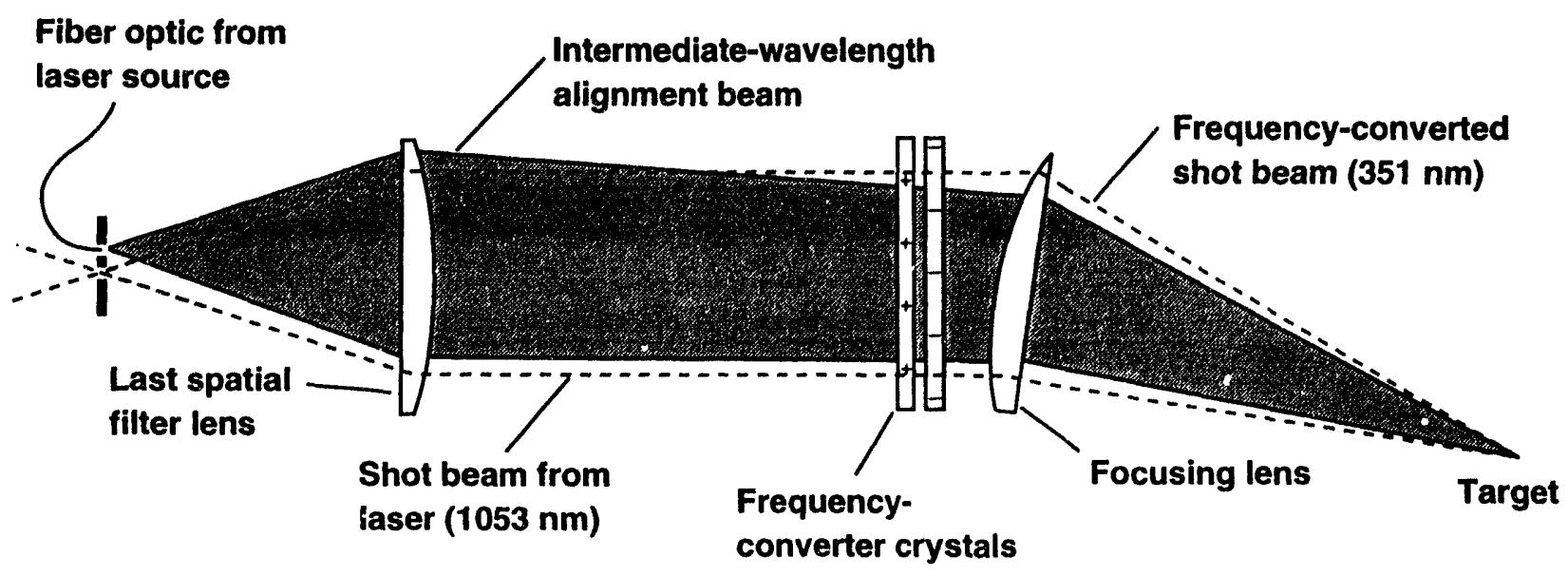

Figure 4. An intermediate-wavelength alignment beam focuses on the target at the same position as the $351-\mathrm{nm}$ shot beam. 

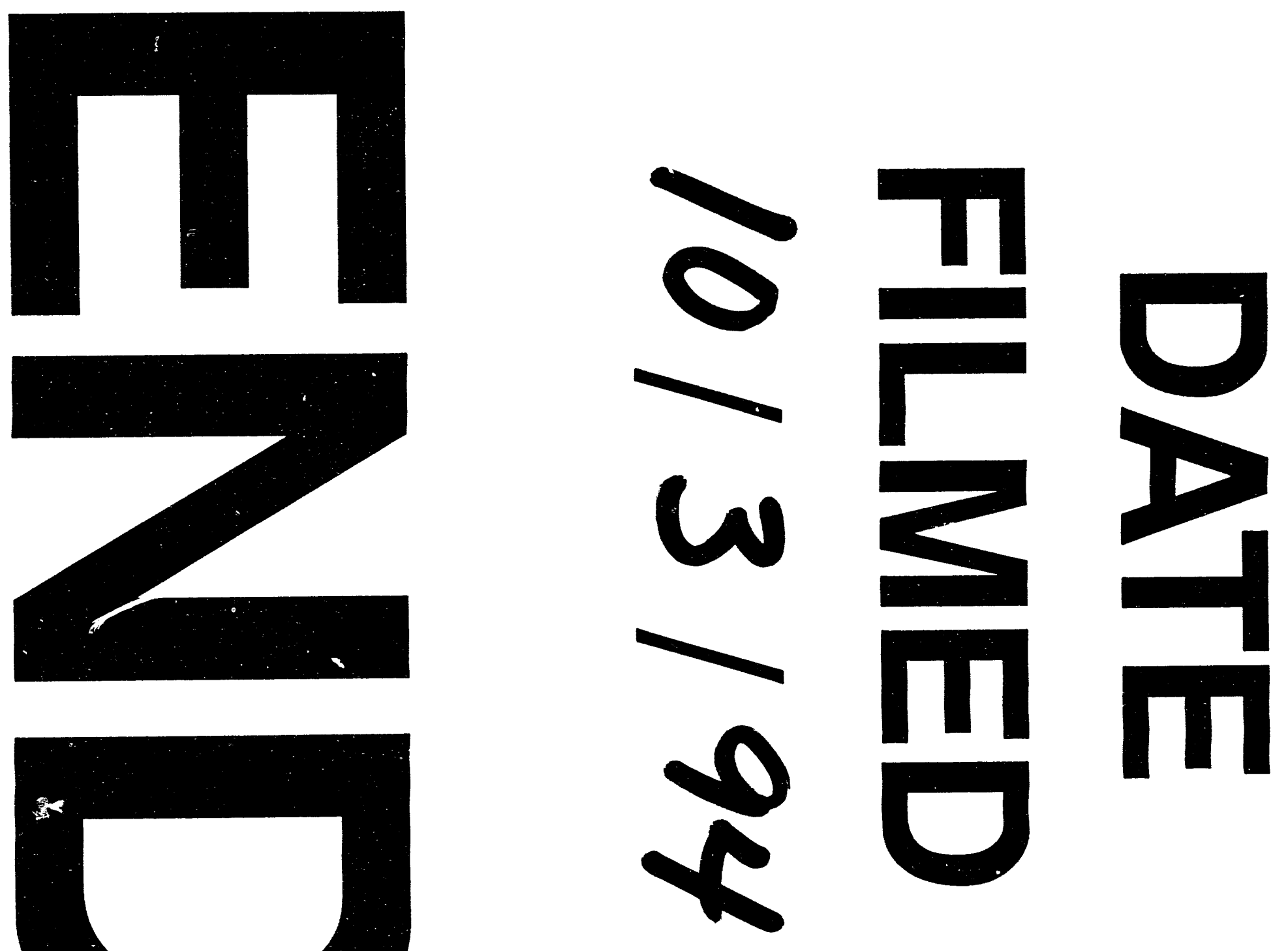
